\title{
Propuesta de Formulación, Implementación y Evaluación de una Política Pública para Reducir los Residuos Sólidos en la Ciudad de MÉXICO
}

\author{
Pablo Gerardo Ríos Zertuche Diez ${ }^{1}$
}

\author{
${ }^{1}$ Instituto Tecnológico de Tlalpan \\ Autorpara correspondência: pablogerardo.rios@gmail.com
}

Recebido em 18 de dezembro de 2018. Aceito em 19 de dezembro de 2018. Publicado em 28 de dezembro de 2018.

\begin{abstract}
Resumen - Ante la gran cantidad de residuos sólidos que diariamente se generan en la Ciudad de México, se deben encontrar soluciones que tengan mayor impacto en su reducción. El problema se ha vuelto sumamente difícil de resolver. Ante esto, es necesario formular una política pública que verdaderamente ayude a solventar el problema. Para ello se utilizó la metodología para la Formulación, Implementación y Evaluación de una Política Pública del Manual de Análisis y Diseño de Políticas Públicas, con la cual se llegó a una propuesta más sólida que contribuiría de manera más firme a tener mayores recursos para el manejo de los residuos sólidos o bien a disminuirlos de manera importante. Se encontró que aunque los consumidores ven como algo práctico e higiénico el uso de empaques y desechables, son las empresas las que los producen y ofrecen, por lo tanto, al hacer una política pública que incentive a los fabricantes a eliminar su producción de empaques y envases contaminantes, y a los comercios y entidades de servicio a no utilizar bolsas ni desechables, se tendrán grandes posibilidades de disminuir los desechos sólidos.
\end{abstract}

Palabras Clave: Políticas públicas, desechos sólidos, Reúso, reciclaje.

Proposta para Formulação, Implementação e Avaliação de uma Política Pública para Reduzir os Resíduos Sólidos na Cidade do MéXico

Resumo - Dada a grande quantidade de resíduos sólidos que são gerados todos os dias na Cidade do México, soluções para um maior impacto na sua redução devem ser encontradas. O problema tornou-se extremamente difícil de resolver. Diante disso, é necessário formular uma política pública que realmente ajude a resolver o problema. Com base na proposta metodológica do "Manual de Análise e Elaboração de Políticas Públicas" para formulação, implementação e avaliação de políticas públicas, com a qual chegou-se a uma proposta mais consistente que contribuiria mais firmemente para obter maiores recursos para a gestão dos resíduos sólidos ou reduzi-los significativamente. Os resultados mostram que, embora os consumidores considerem o uso de embalagens e descartáveis como práticas e higiênicas, o alvo para políticas de redução de desperdício devem ser as empresas que produzem esses materiais. Portanto, a formulação de uma política pública que incentive os fabricantes a eliminar a produção de embalagens e recipientes poluentes e estimule o comércio e prestadoras de serviços a não usar sacolas plásticas ou descartáveis poluentes teremos grandes chances de redução de resíduos sólidos.

Palavras-Chave: Políticas públicas, resíduos sólidos, reutilização, reciclagem.

Proposal for the Formulation, Implementation and Evaluation of a Public Policy to Reduce Solid Waste in Mexico City

Abstract - Given the large amount of solid waste that is generated every day in Mexico City, solutions towards a greater impact on its reduction must be found. The problem has become extremely difficult to solve. Given this situation, it is 
necessary to formulate a public policy that truly helps to solve the problem. Based on the methodological proposal of the "Manual of Analysis and Preparation of Public Policies" for the formulation, implementation and evaluation of public policy, I hereby made a policy proposal for the reduction of solid waste. The purpose is to firmly contribute to either increase resources for better management of solid waste or to significantly reduce them. Results show that while consumers perceive the use of packaging and disposables as practical and hygienic, the target for waste reduction policies should be the companies that produce such materials. Therefore, the formulation of a public policy that encourages manufacturers to eliminate the production of contaminating packaging and containers, and stimulate businesses and service entities not to use plastic or disposables bags, increases the possibilities of reducing solid waste.

KeYwords: Public POLICIES, SOLID WASTE, REUSE, RECYCLING.

\section{INTRODUCCIÓN}

La generación de residuos sólidos en la Ciudad de México es un problema que va en aumento. De acuerdo a la Secretaría de Medio Ambiente y Recursos Naturales (Notimex 2018), cada persona genera $1.2 \mathrm{Kg}$. de basura al día, y aunque desde el año 2000, la población de la ciudad no ha crecido de manera importante, la de la Zona Metropolitana del Valle de México si lo ha hecho, y de ahí diariamente se desplazan a esta ciudad un millón setecientos veinte mil personas a trabajar o estudiar (Navarro 2015), generando basura, esto ha provocado un incremento que se refleja así “...para 2017 la cantidad de basura generada al día pasó a 12 mil 998 toneladas, esto es, cuatro millones 744 mil 270 millones de toneladas de residuos sólidos anuales. La diferencia entre 2012 y 2017 es de 94 mil 170 toneladas" (Díaz 2018).

Por otro lado, como la sociedad se ha vuelto más consumista (Redacciones 2018), ha habido un fuerte incremento en la generación de residuos urbanos. Los envases de refresco y leche, por ejemplo, antes eran de vidrio y se canjeaban en las tiendas, descontándose el importe de la botella. En la actualidad la mayoría son desechables, y a pesar de que se han diseñado nuevas formas de empaque, éstas siguen siendo contaminantes.

Una de las problemáticas adicionales es que no se alcanza a recolectar toda la basura, lo que genera que parte de ésta quede en las calles, lo que a su vez propicia la obstrucción de coladeras, lo que puede provocar inundaciones u otros problemas de tipo sanitario. Para agravar esta situación, existe una baja educación ambiental en importantes sectores de la población, tomando con desinterés el tema.

La problemática continúa, pues posteriormente a su colecta, la basura se transfiere a basureros de cielo abierto del Estado de México y Morelos, creado contaminación, así como problemas de higiene y fauna nociva.

En este trabajo se considera que se deben crear políticas públicas que disminuyan la alta generación de residuos sólidos y que permitan la existencia de más recursos para su manejo, determinando cuál política tendría un mayor impacto en ello.

Para lograrlo, se siguió la metodología del Manual de Análisis y Diseño de Políticas Públicas (OrdóñezMatamoros 2013), razón por la cual, las secciones en las que se divide el presente trabajo, son acordes al ciclo del análisis y diseño de políticas públicas establecido en dicho manual.

El trabajo comienza poniendo sobre la mesa la problemática de la basura en la Ciudad de México y explica el ciclo para la creación, monitoreo y evaluación de una política pública.

Inicia con el apartado "verifique, defina y detalle el problema", donde, a través de un árbol de problemas se determina la causalidad de éste, lo que proporciona bases para entender mejor la problemática de la basura.

Posteriormente, se identifican los criterios de decisión, con los que se fijan las bases para seleccionar la mejor solución; después se evalúan las alternativas y se recomienda la implantación de la mejor solución, para finalmente planear y proponer su implementación.

Por último se incluyen los apartados de monitoreo de la política pública y evaluación del impacto, en los que se establecen los lineamientos generales para dar seguimiento y evaluar el funcionamiento de la política pública 
El objetivo del presente trabajo es el de proponer una política pública que ayude a reducir los residuos sólidos en la Ciudad de México y por lo tanto a facilitar su manejo y disminuir la problemática en su conjunto. Dada su relevancia, esta política podría extenderse a nivel internacional, propiciando un mejor medio ambiente.

\section{¿QUÉ SON LAS POLÍtTICAS PÚBLICAS?}

Gonzalo Ordoñez Matamoros y los otros autores del libro Manual de Análisis y Diseño de Políticas Públicas, afirman que:

Una de las mayores dificultades para comprender el término "política pública" radica en que se trata de un concepto que en la práctica no existe en castellano. En efecto, el concepto de "policy" es tradicionalmente traducido como política, lo que a su vez suele ser interpretado como "politics" (la actividad política como competencia por el poder). De esta manera, se confunde el arte de la puja por el poder ("politics") con lo que hoy se conoce como política pública, el arte de saber qué se hace con el poder una vez este es adquirido.

Política pública es el conjunto de acciones implementadas en el marco de planes y programas gubernamentales diseñados por ejercicios analíticos de algún grado de formalidad, en donde el conocimiento, aunado a la voluntad política y los recursos disponibles, viabilizan el logro de los objetivos sociales (Ordóñez-Matamoros et al. 2013, p. 32).

Es muy importante no caer en el error de tercer tipo, que implica ofrecer una solución al problema que en realidad no lo es; es decir, buscar solucionar el problema equivocado. Esto sucede cuando se le da demasiada importancia a la solución y no suficiente al análisis del problema en sí, asimismo a olvidar que los problemas evolucionan y a no considerar que los problemas vienen en conjunto.

Por otra parte, el artífice de las políticas públicas es el analista, que juega un papel preponderante en el conocimiento y suministro de la información relevante de dichas políticas, coadyuvando a que la sociedad logre los objetivos que pretende alcanzar de la mejor forma posible.

\section{El Análisis y Diseño de Políticas Públicas}

Este es un ciclo de ocho pasos para diseñar políticas públicas con una metodología clara que permite descubrir el problema y adentrarse en él, investigar sus causas, evaluar las alternativas de solución y seleccionar la más adecuada; para posteriormente implementarla, darle seguimiento y evaluar su impacto.

Los pasos son (Figura 1):

1. Verifique, defina y detalle el problema.

2. Identifique los criterios de decisión

3. Identifique las alternativas de selección

4. Evalúe las alternativas

5. Recomiende la implementación de la mejor solución.

6. Planifique la implementación

7. Monitoree la política implementada

8. Evalúe el impacto

Todo esto hace que se tome con mayor seriedad y con bases más firmes la formulación de políticas públicas, haciendo un análisis del problema para evitar el error de tipo tres, y enfocando los esfuerzos para que la política pública impacte, y planeando la evaluación y seguimiento de la política. 
Figura 1. El Análisis y Diseño de Políticas Públicas.

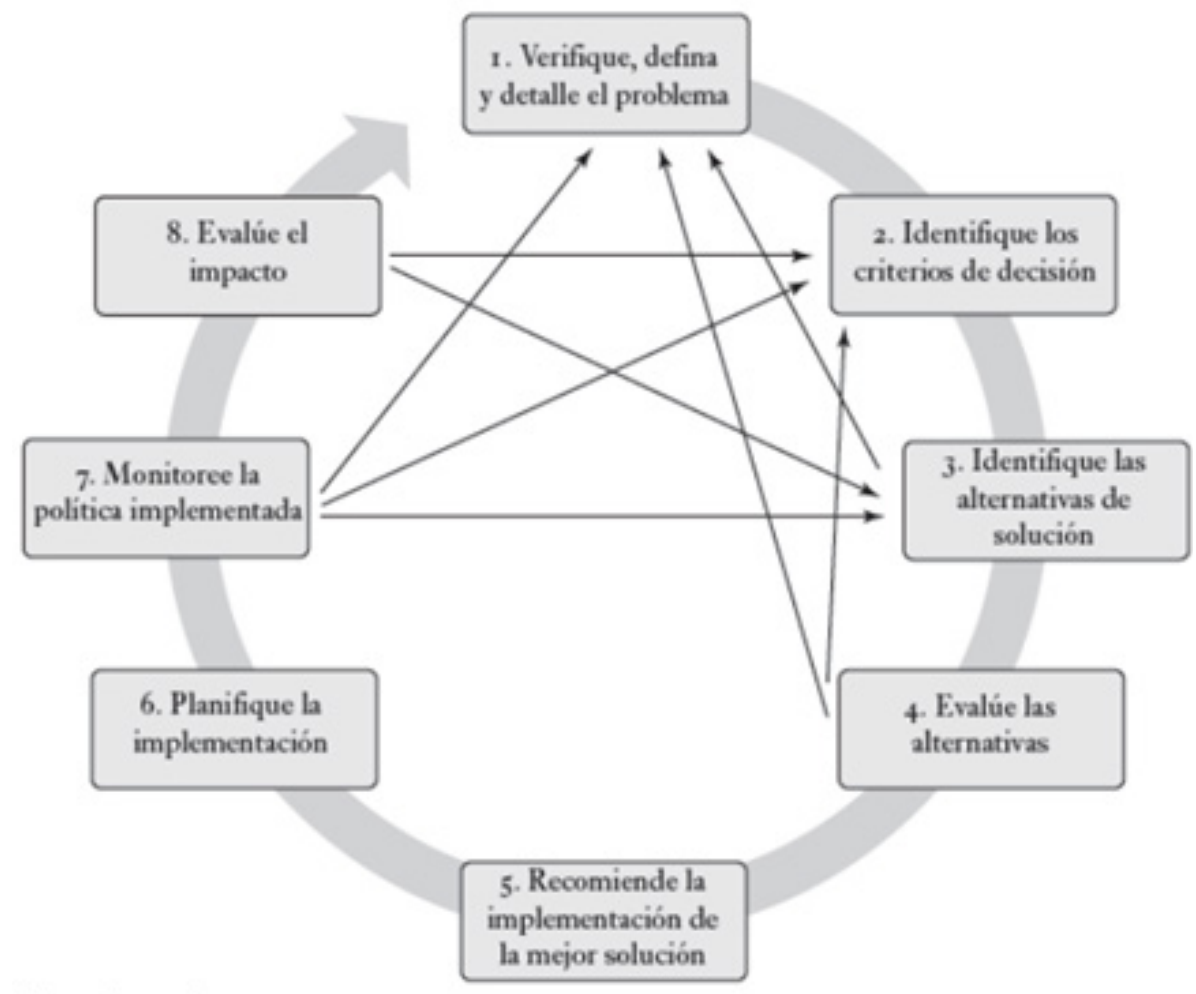

Fuente: Autores del Manual de Análisis y Diseño de Políticas Públicas (Ordóñez-Matamoros 2013).

\section{La Basura en la Ciudad de México}

El artículo "Ciudad de México, al borde del colapso por la generación excesiva de basura” (Reveles 2018), cita "El principal problema de Ciudad de México no es la separación y el reciclaje, sino la excesiva generación de basura." Y afirma que "En 2016, de acuerdo con el Inventario de Residuos Sólidos, la capital del país producía 12 mil 920 toneladas de basura al día."

De acuerdo al estudio "El problema de la basura en la Ciudad de México" (Mora Reyes 2004), indica que "en las sociedades preindustriales, tanto el volumen relativamente limitado de generación, como la composición predominantemente orgánica de muchos de los residuos o la biodegradabilidad de éstos, el manejo de los mismos estuvo limitado en el mejor de los casos, a llevarlos a un sitio distante de las comunidades para ser enterrado a los ciclos de la vida." Sin embargo, continúa el informe, con la industrialización se han acelerado los procesos de producción y se incrementó fuertemente el consumo de los bienes producidos, y con ellos se produjo una gran cantidad de envases y embalajes, generándose, por lo tanto, enormes volúmenes de residuos.

Debido a los cambios sociales referidos, el consumidor se ha acostumbrado cada vez más a envases desechables. Incluso la higiene se percibe como un producto envuelto en plástico, el agua viene en una botella (PET), las hamburguesas en una caja de unicel, los pastelitos empacados en plástico y un papel metalizado; produciendo con ello gran cantidad de residuos.

Otros productos tienen una obsolescencia programada, que es la reducción deliberada de la vida útil de un producto para incrementar su consumo por parte del fabricante (Sostenibilidad para Todos 2017), esta obsolescencia programada provoca que "cada año se generen hasta cincuenta millones de toneladas de residuos electrónicos que en un altísimo porcentaje -en torno al 85 \%- son desechados, sin ningún control y terminan en vertederos de países en desarrollo, lo que supone un riesgo para el entorno y para la salud de personas, animales y plantas." Vivimos en la era del consumismo, ahora exacerbada por el neoliberalismo; dispositivos electrónicos, autos, aparatos electrodomésticos, se vuelven desechables al paso del tiempo y conviene más comprar uno nuevo que reparar el que ya se tiene. Todo eso va a parar a la basura (Figura 2). 
Figura 2. Generación de Basura por Tipo de Fuente.

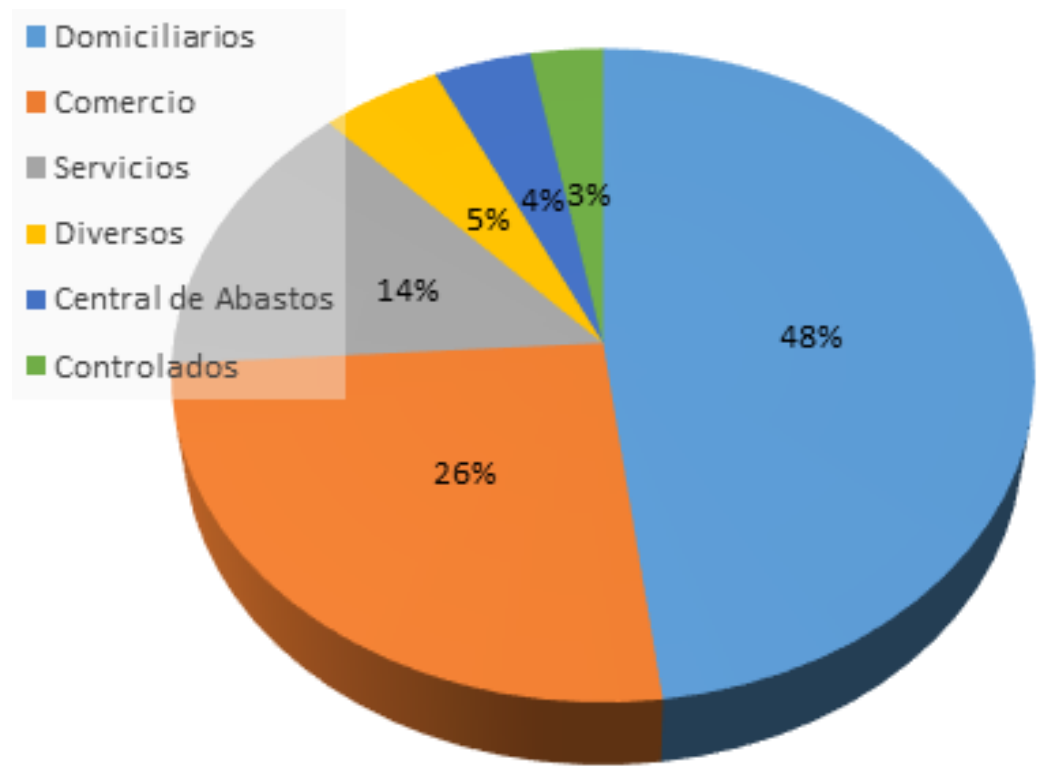

Fuente: Elaboración propia de datos de Inventario de Residuos Sólidos, Secretaría del Medio Ambiente del Gobierno de la Ciudad de México 2017).

Casi la mitad de la basura de la Ciudad de México proviene de casas, departamentos y unidades habitacionales (48\%), los comercios y servicios representan el 26\% y 14\% respectivamente. En el 2015, en la Ciudad de México había 2’599,081 viviendas particulares (INEGI 2015), habrá que imaginar la infraestructura y la gestión necesarias para recoger la basura de todas ellas, que están dispersas a lo largo de 1,485 km2, que es la superficie de esta Ciudad.

Los desechos sólidos controlados, que son los que provienen de unidades médicas, laboratorios, veterinarias, terminales terrestres, de transporte aéreo y centros de readaptación social representan únicamente el 4\%, pero su manejo es delicado y no hay información clara de que siempre se sigan los procedimientos acordes a las normas.

\section{Tipos de Basura}

Dependiendo del tipo de basura que se genere, se pueden diseñar acciones específicas para disminuir la problemática. Existe la posibilidad de hacer composta, reusar o reciclar, pero existe un 32.6\% no identificada (Mora Reyes 2004), lo que no nos permite asegurar la composición de la basura en su totalidad.

En cuanto a la basura identificada, la mayor parte son residuos alimenticios que representan el $31 \%$; en segundo lugar, papel y cartón con el 14.2\%; 9.8\% de desechos de jardinería y el 6.6\% de vidrio (Figura 3). 
Figura 3. Tipos de basura en la Ciudad de México.

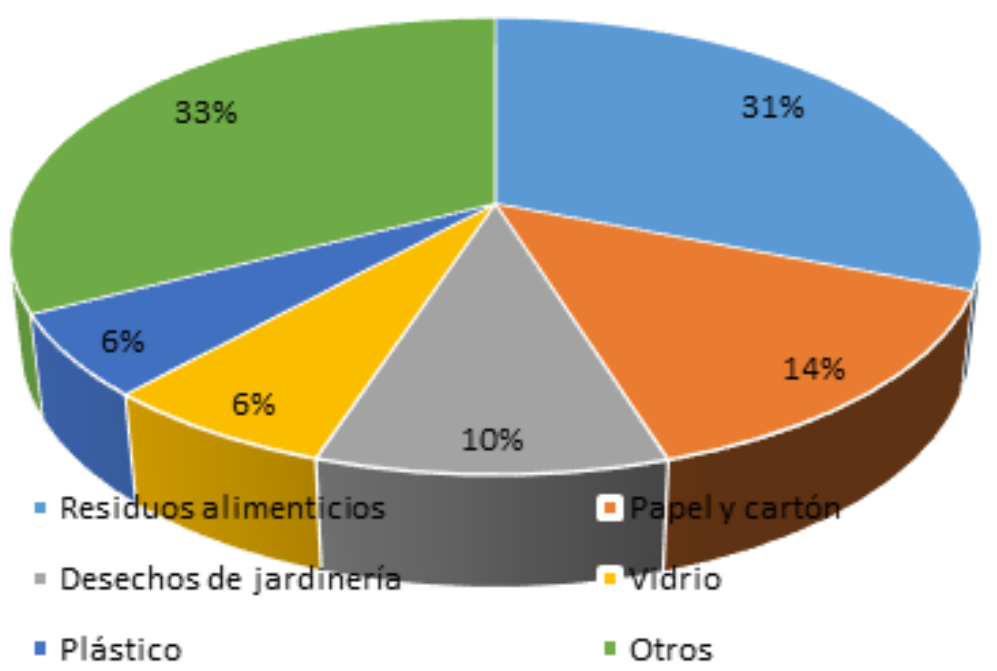

Fuente: Elaboración propia de datos de Mora Reyes; El problema de la basura en la Ciudad de México (2004)

\section{Destino DE LOS DESEChOS SÓLIDOS}

Inicialmente, los residuos sólidos son recogidos por camiones de basura y llevados a alguna de las doce estaciones de transferencia, de ahí se mandan a la planta de composta, la planta de selección o a los lugares de disposición final. Los porcentajes reportados se pueden observar en la Tabla 1.

Tabla 1. Destino de los desechos sólidos.

\begin{tabular}{|l|c|c|c|c|}
\hline País & Relleno Sanitario & Incineración & Composteo & Reciclaje \\
\hline Estados Unidos & 73 & 14 & 1 & 12 \\
\hline Japón & 27 & 25 & 2 & 46 \\
\hline Alemania & 52 & 30 & 3 & 15 \\
\hline Francia & 48 & 40 & 10 & 2 \\
\hline Suecia & 40 & 52 & 15 & 3 \\
\hline México & 94 & --- & --- & 6 al 10 \\
\hline
\end{tabular}

Fuente: Inventario de residuos sólidos página 28 (Mejía Vargas, A. C. (coordinador) 2017)

Manifiesta la Secretaría del Medio Ambiente del Gobierno de la Ciudad de México: (Mejía Vargas, A. C. (coordinador) 2016).

Los residuos que por sus características no pudieron reincorporarse a procesos productivos son enviados a Rellenos Sanitarios, que son obras de infraestructura que aplican métodos de ingeniería para la disposición final de los residuos sólidos, minimizando con ello los riesgos por contaminación al ambiente y a la salud.

Actualmente la Ciudad de México no cuenta con un espacio donde disponer sus residuos, por lo que estos son enviados a cuatro rellenos ubicados en el Estado de México: 51\% a la Cañada, 38\% Milagro, 5\% Cuautitlán, 3\% Chicoloapan y uno en Cuautla, Morelos que recibe el 3\% (Figura 4). 
Figura 4. Disposición de los Desechos Sólidos en México.

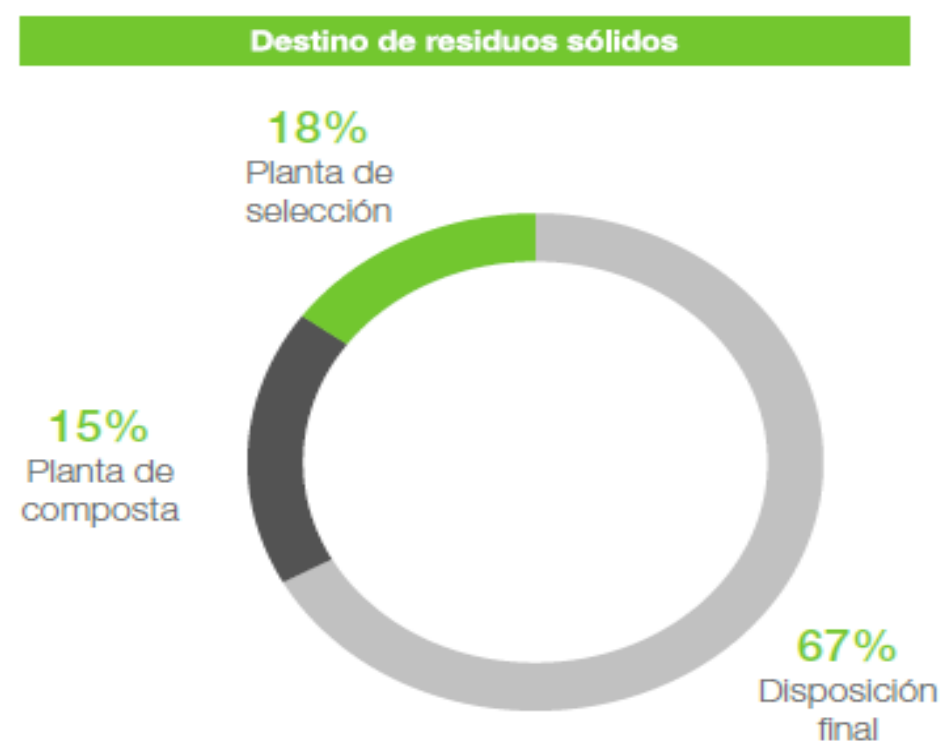

Fuente. Sancho y Cervera J. Rosiles GG. Situación del Manejo Integral de los Residuos Sólidos en México. Sedesol. 1999, en Cortinas, Cristina. Hacia un México sin Basura. Bases y aplicaciones de las legislaciones sobre residuos. PVEM. 2001. Página 326.

\section{Verificando, Detallando y Definiendo el Problema}

En las secciones anteriores se ha dado a conocer el grave problema que representa la basura para la Ciudad de México, en esta sección se acotará el problema, dando pie para iniciar el análisis del mismo y con ello comenzar a utilizar la metodología del Análisis y Diseño de Políticas Públicas.

El problema se puede definir como:

Diariamente se produce una gran cantidad de basura en la Ciudad de México (alrededor de 13 mil toneladas). El gobierno hace un esfuerzo importante, pero insuficiente para su disposición. Las tendencias marcan que la problemática se va a agravar.

Para determinar más claramente las causas del problema se utilizará el árbol de problemas.

Debido a problemas de espacio se separará el árbol de problemas en tres secciones (Figura 5), que llevan al mismo problema: Exceso de basura en la Ciudad de México; esto para que el tamaño de tipografía sea legible. 
Figura 5. Árbol de Problemas de los Desechos Sólidos de la Ciudad de México.

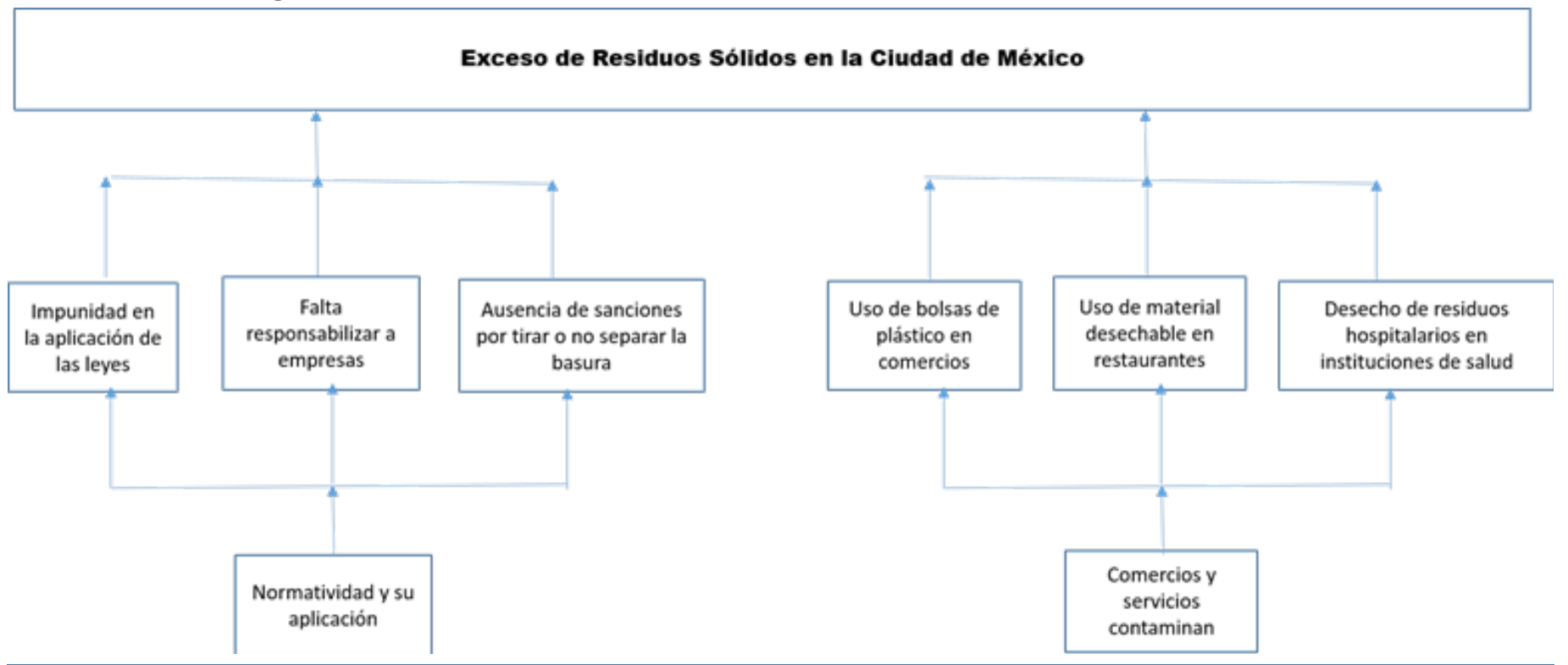

Exceso de Residuos Solidos en la Ciudad de México

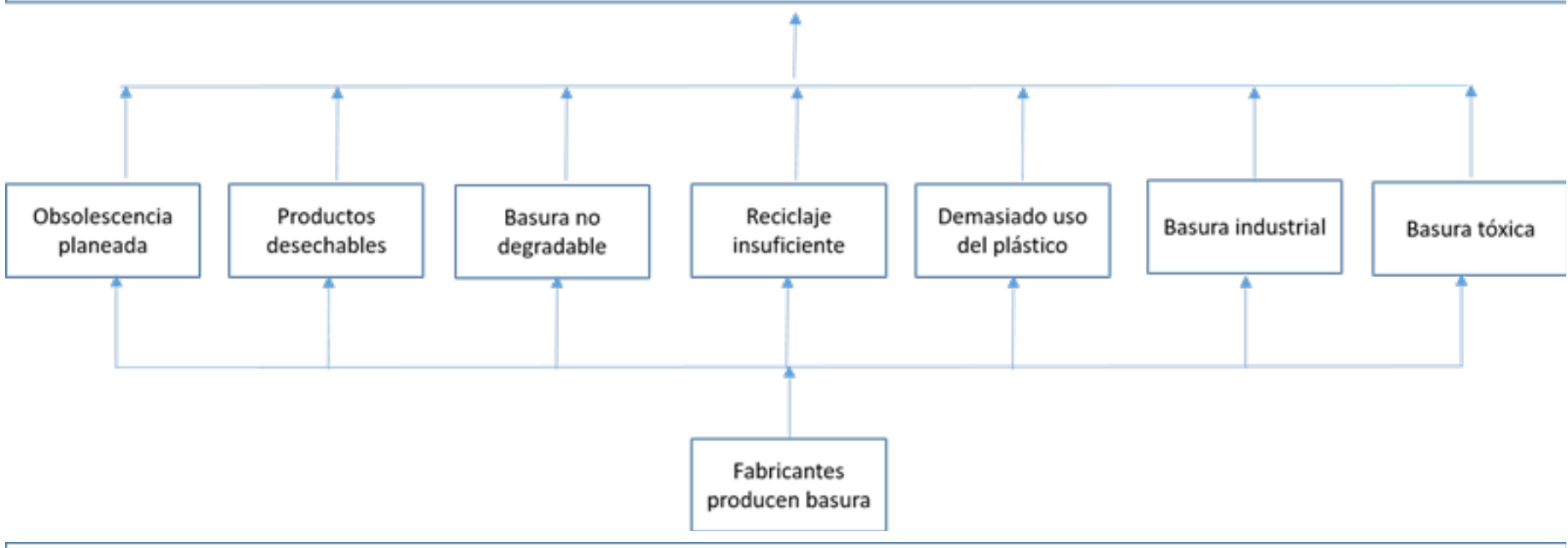

Exceso de Residuos Solidos en la Ciudad de México

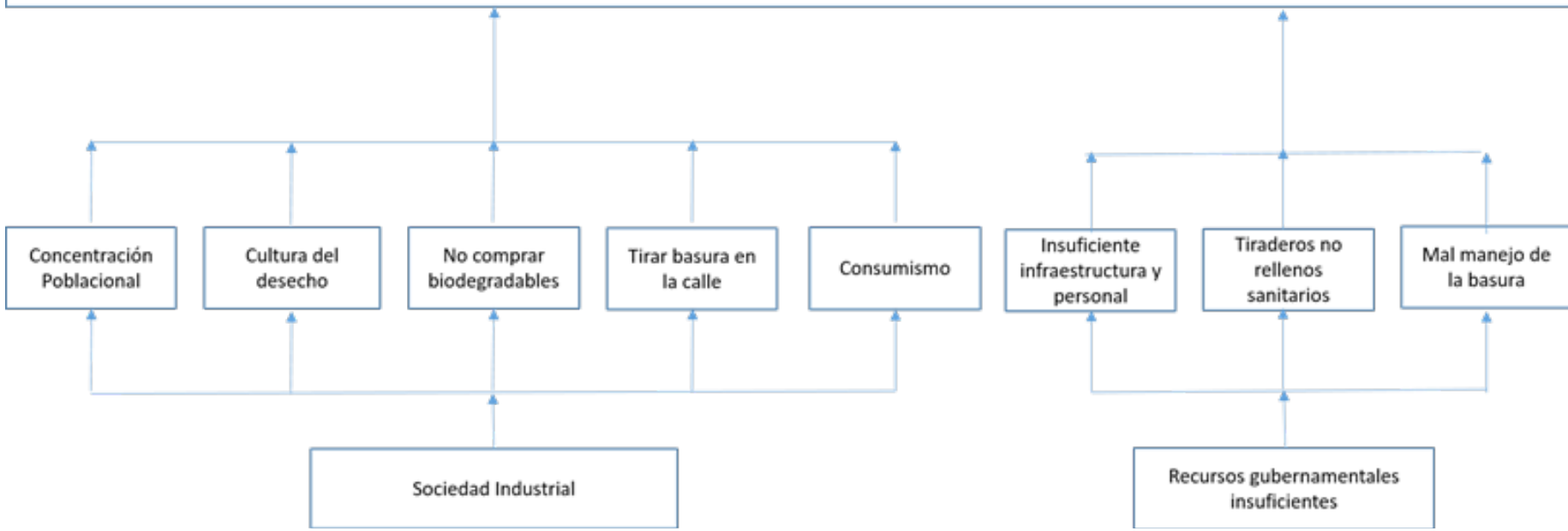

Se puede observar en el árbol de problemas que se dividieron en cinco a las causales del exceso de basura, estas son: sociedad industrial, recursos gubernamentales insuficientes, normatividad y su aplicación, fabricantes que producen basura y comercios y servicios que contaminan. De acuerdo a lo que se pudo observar, los principales generadores de basura inorgánica son los consumidores, debido a que han adquirido una cultura consumista y de desecho, pero las empresas, son las que manufacturan estos productos para satisfacer al cliente; por ejemplo, entre 
las mayores generadoras de plástico según Greenpeace están Coca Cola, PepsiCo, Nestlé y Danone. (Huffpost 2018).

\section{IDENTIFIQUE LOS CRITERIOS DE DECISIÓN}

El segundo paso del análisis es el de identificar los criterios de decisión. Los cuatro criterios más comúnmente utilizados (Ordóñez-Matamoros 2013) son:

1. Viabilidad técnica

2. Viabilidad política

3. Viabilidad administrativa

4. Viabilidad económica y financiera

Para efecto de esta decisión se tomarán en cuenta sobre todo la viabilidad política y la económica y financiera, porque es imprescindible contar con los recursos necesarios para aplicar la política que se establecerá; además se podrán tomar en consideración criterios como efectividad, eficiencia, suficiencia, equidad, capacidad de respuesta y apropiado para la sociedad.

\section{IDENTIFICACIÓN DE ALTERNATIVAS DE SOLUCIÓN}

Se utilizarían, de acuerdo a Weimer y Vining (Ordóñez-Matamoros 2013) políticas públicas para establecer normativas e imponer impuestos para desincentivar el uso de empaques de plástico o contaminantes e incrementar el reúso de estos. Para ello modificar las leyes pertinentes.

Las alternativas serían, por lo tanto,

1. Una iniciativa de ley hacia las empresas, que:

a) Cobre un impuesto adicional a las empresas que generen productos desechables o empaques y embalajes, acorde a la cantidad de basura que generen o las obligue a que sean ellas las que recojan esa basura.

b) Obligue a las empresas electrónicas y químicas de consumo (fabricantes de baterías, por ejemplo) a recoger y reprocesar sus productos al ser desechados.

c) Se obligue a que se imprima un código de recuperación en cada envase o empaque para monitorear el reciclaje de este; si en tres meses no se recupera; multar a la empresa.

d) Dedicar los fondos recolectados al manejo de los residuos sólidos.

2. Una iniciativa de ley hacia el consumidor, que:

a) Cobrar impuestos adicionales por exceso de basura.

b) Fuertes multas por tirar basura en la calle o por no separar basura.

c) Impuestos para desincentivar la compra de envases y empaques plásticos.

3. Una mezcla de las anteriores opciones.

\section{Evaluación DE ALTERNATIVAS}

Para seleccionar la mejor alternativa, se utilizó el Método de Evaluación por Calificación Básica, que consiste en evaluar lo más objetivamente los criterios anteriormente propuestos, pero de manera cualitativa, poniendo 
una A de aprobado cuando cumple con el criterio, siendo la mejor opción la que tenga el mayor número de A. Se califica como R cuando la alternativa reprueba dicho criterio y se establece NA cuando no aplica. Se consideraron tres alternativas (Tabla 2).

Para la primera opción procedería a aplicar impuestos y a normar a las grandes empresas transnacionales como Coca Cola, por lo que se esperaría, un fuerte rechazo a la normatividad, por otro lado, se requeriría que previamente se hiciera una campaña de rechazo a la "basura que acompaña tu producto", para concientizar al consumidor del daño ecológico que causan estos envases. Además, se requeriría apoyo de organismos internacionales como 4Oceans y Greenpeace para que la presión sea internacional.

El problema principal sería la reacción de las grandes empresas y su poder de cabildeo y corrupción en los distintos órdenes de gobierno. Sin embargo, si llegará a aplicarse cabalmente la ley, terminaría inmediatamente con el incremento de basura en la Ciudad de México y en el mediano plazo disminuiría, haciendo más fácil su gestión.

La segunda opción también es buena, pues es necesario educar al consumidor para que no demande empaques ni bolsas, ponga la basura en su lugar y la separe.

En cuanto a la última, en la cual se toman en consideración los postulados de las dos alternativas anteriores, podría ser una buena opción también.

Tabla 2. Método de Evaluación por Calificación Básica

\begin{tabular}{|l|c|c|c|c|c|c|c|c|}
\hline Alternativa & $\begin{array}{c}\text { Viabilidad } \\
\text { Política }\end{array}$ & $\begin{array}{c}\text { Viabilidad } \\
\text { Económica }\end{array}$ & Efectividad & Eficiencia & Suficiencia & $\begin{array}{c}\text { Capacidad } \\
\text { de respuesta }\end{array}$ & $\begin{array}{c}\text { Apropiado } \\
\text { para la } \\
\text { sociedad }\end{array}$ & Total \\
\hline Consumidores & $\mathrm{A}$ & $\mathrm{A}$ & $\mathrm{R}$ & $\mathrm{R}$ & $\mathrm{A}$ & $\mathrm{R}$ & $\mathrm{A}$ & $4 \mathrm{~A}$ \\
\hline Empresas & $\mathrm{A}$ & $\mathrm{A}$ & $\mathrm{A}$ & $\mathrm{A}$ & $\mathrm{A}$ & $\mathrm{A}$ & $\mathrm{A}$ & $7 \mathrm{~A}$ \\
\hline Mixto & $\mathrm{A}$ & $\mathrm{A}$ & $\mathrm{R}$ & $\mathrm{R}$ & $\mathrm{A}$ & $\mathrm{R}$ & $\mathrm{A}$ & $4 \mathrm{~A}$ \\
\hline
\end{tabular}

Fuente: Elaboración propia en base al Manual de análisis y diseño de políticas públicas (Ordóñez-Matamoros 2013)

De acuerdo a este método se seleccionó la alternativa "empresas", por ser la que mayor grado de aprobación de las distintas variables tuvo.

\section{RECOMENDANDO LA IMPLEMENTACIÓN DE LA MEJOR SOLUCIÓN}

Las verdaderas generadoras del problema de la basura son las empresas que manufacturan productos desechables, en conjunto con abundantes empaques y embalajes. La población no solo se ha acostumbrado a ello, sino que ahora lo ve como una necesidad. La normatividad que se establezca debe abarcar a toda la industria. Por ejemplo, si ninguna refresquera ofreciera envases PET o cualquier desechable, si no se permitiera la importación de estos, y se hiciera una campaña de concientización en escuelas, oficinas de gobierno, redes sociales y televisión para terminar con el uso de estos envases, seguramente repercutiría en la cantidad de basura generada.

Por otro lado, se debe obligar a las empresas a que administren mejor la obsolescencia programada y se hagan cargo de sus productos al ser desechados. Lo mismo para empresas químicas, como para fabricantes de aceites para autos, baterías de litio o alcalinas, de la misma manera que para fabricantes de equipos electrónicos.

$\mathrm{Al}$ principio la restricción o el cobro de impuestos repercutiría en el bolsillo del consumidor, pero en el mediano plazo las compañías encontrarán mejores opciones para fabricar y envasar sus productos. 


\section{DisEÑO DE UN PLAN DE IMPLEMENTACIÓN}

El objetivo final debe ser: Presentar una iniciativa de reforma a la Ley General para la Prevención y Gestión Integral de Residuo (Cámara de Diputados del H. Congreso de la Unión 2003) para que sea aprobada con el fin de que se disminuya la generación de envases y empaques en productos fabricados en México o importados a nuestro país; así como la utilización de bolsas y material desechable en comercios y servicios.

Requisitos previos:

- Crear una campaña de concientización en medios para que la población tenga una actitud negativa hacia la basura que acompaña a los productos. No a envases de plástico, ni ningún elemento que no sea biodegradable.

- Realizar un cabildeo en las Cámaras de Diputados y Senadores hasta asegurar una mayoría para la aprobación.

- Hablar con los Directores Generales de las empresas que más generen basura para negociar la entrada de la ley y no inviertan en nuevas máquinas que vayan a crear más desechos sólidos, sino en materiales biodegradables.

- Buscar con ellos alternativas de empaque, asesorarse de científicos de instituciones de educación superior.

Dentro de esta iniciativa se deben sentar las bases para cobrar mayores impuestos a las empresas generadoras de basura, lo que ayudará a contar con más recursos para fomentar un mejor manejo de los residuos sólidos y a desincentivar a las mayores generadoras de basura a seguir contaminando.

Todo esto puede tardar un sexenio para dejar un escenario plausible. Una vez realizado esto; crear la iniciativa a nivel nacional, tratando de hablar con secretarios de estado y de ser posible con el presidente para afianzar su aprobación. Se requiere un equipo de al menos cincuenta personas y un presupuesto significativo para lograrlo.

En la tabla 3 se propone un cronograma para la implementación que va desde el cabildeo entre partidos para crear un ambiente propicio a la aprobación de la ley, hasta la aprobación misma. Cada número significa trimestres, con lo que se estaría contemplando un año para llevar a cabo el proyecto.

Tabla 3. Cronograma para la implementación de la política pública

\begin{tabular}{|c|c|c|c|c|c|c|c|c|c|c|c|c|c|}
\hline Actividad & Responsable & 1 & 2 & 3 & 4 & 5 & 6 & 7 & 8 & 9 & 10 & 11 & 12 \\
\hline $\begin{array}{c}\text { Cabildeo y } \\
\text { negociación inicial }\end{array}$ & Director & \multicolumn{2}{|c|}{} \\
\hline $\begin{array}{c}\text { Creación de } \\
\text { iniciativa de ley }\end{array}$ & Jurídico & & & & & & & & & & & & \\
\hline $\begin{array}{c}\text { Revisión y } \\
\text { renegociación }\end{array}$ & Director & & & & & & & & & & \\
\hline Campaña de apoyo & Comunicación & & & & & & & & & & \\
\hline $\begin{array}{c}\text { Aprobación de la } \\
\text { ley }\end{array}$ & Director & & & & & & & & & & & \\
\hline
\end{tabular}

\section{MONITOREO DE LA POLÍTICA IMPLEMENTADA}

Se deberá monitorear diariamente la basura en cada una de las estaciones de transferencia para verificar su peso y composición, además se deberán supervisar dieciséis puntos de los más transitados de la ciudad; uno por cada delegación, para confirmar la limpieza de las calles.

Así mismo se dará seguimiento al aumento o disminución en el gasto de basura, para medir el costo beneficio.

Se espera un decremento de hasta un 30\% al término del primer año en los niveles de plástico que se encuentran en la basura. Se realizará una evaluación ex ante para probar los indicadores de los que se habla en la sección siguiente y conocer la situación que detenta actualmente la situación de los residuos sólidos en la Ciudad de México, de acuerdo a los indicadores. 


\section{EVALUAR EL IMPACTO}

Para la evaluación se crearán cinco indicadores que permitirán comprobar los avances que tendrá esta política pública.

Los indicadores son:

1. Indicador del peso de la basura: Éste se obtiene tomando el peso diario de la basura procesada en las estaciones de transferencia. Se tomará como cien el del día del inicio del programa y cada día tendrá un índice mayor o menor a cien de acuerdo al incremento o decremento de los residuos sólidos. El indicador debe tener ciclos porque por ejemplo en navidad se incrementa la basura.

2. Indicador de composta: Este indicador medirá qué porcentaje de la basura puede ser usado como composta y deberá aumentar a medida que se elimine el plástico.

3. Indicador de reúso: Porcentaje de la basura que puede volver a reutilizarse, como las botellas de vidrio. Este debe disminuir porque se deben usar otras vías para el reúso y no tirar a la basura los envases.

4. Indicador de reciclaje: Porcentaje de la basura que puede reciclarse como el cartón y el PET. Deberá disminuir cediendo el paso a la composta.

5. Indicador de facilidad de separación: Si se pone en marcha el programa y verdaderamente se separa la basura se logrará que los materiales reutilizables sean seleccionados en mucho menor tiempo y requiriendo menos personal que actualmente.

Con estos indicadores se podrá evaluar si se está avanzando con esta política pública o si por el contrario existiera un retroceso en el procesamiento de los residuos sólidos.

\section{Conclusiones}

La basura es un problema que va en aumento y se deben tomar medidas que lo disminuyan. La metodología del Análisis y Diseño de Políticas Públicas permite hacer un análisis que aclara con mayor precisión las causas de este problema, ayudando a encontrar la solución más idónea.

Como muchos de los problemas que aquejan a nuestro país, el de los residuos sólidos es muy complejo, pues involucra a muchos actores en él. Las empresas, el gobierno, los pepenadores, la sociedad en conjunto.

Sin embargo, su solución es urgente y tiene un interés internacional, pues afecta la ecología de todo el planeta.

Con este trabajo se encontraron las principales causas de la generación de residuos sólidos, lo que dirigió la solución hacia la implementación de una política pública que incentive a las empresas a evitar la inclusión de empaques y envases contaminantes. Esto debería terminar en una modificación a las leyes que provoque mayores impuestos y multas a las empresas contaminantes.

La solución encontrada no es sencilla de implementar, pues se enfrenta a poderosas compañías, pero considero que es la que mayor impacto tendría.

Queda este trabajo como semilla para que se establezca una iniciativa de ley que verdaderamente cambie las condiciones de vida de los citadinos y mejore la ecología del lugar, lo cual también impactará a todo el país pues las leyes son federales.

\section{REFERÊNCIAS}

Cámara de Diputados del H. Congreso de la Unión. (2003). Ley General para la Prevención y Gestión Integral de Residuos Sólidos. Ciudad de México: Diario Oficial de la Federación. 
Díaz, O. (2 de septiembre de 2018). Crece basura generada en la Ciudad de México. Obtenido de Publimetro: https:/ / wnw. publimetro.com.mx/mx/noticias/2018/09/02/crece-basura-generada-la-cdmx.btml [Verificado el 18 de diciembre de 2018]

INEGI. (2015). Cuéntame; Información por Entidad. Obtenido de Ciudad de México: http:/ / cuentame.inegi.org.mx/monografias/ informacion / df/poblacion/vivienda.asp $x$ ?tema $=$ mes $=09$ [Verificado el 18 de diciembre de 2018]

Mora Reyes, J. A. (2004). El problema de la basura en la Ciudad de México. Ciudad de México: Fundación de Estudios Urbanos y Metropolitanos Adolfo Christlieb Ibarrola.

Navarro, M. F. (22 de diciembre de 2015). Población flotante: DF centro laboral y escolar del Estado de México. Obtenido de Excélsior: https:/ / wnn.excelsior.com.mx/comunidad/2015/12/24/1065140 [Verificado el 18 de diciembre de 2018]

Notimex. (16 de febrero de 2018). Esta es la cantidad de basura que genera cada mexicano al día. Obtenido de Excélsior: https:// www.excelsior.com.mx/nacional/2018/02/16/1220819 [Verificado el 18 de diciembre de 2018]

Ordónez-Matamoros, G. (2013). Manual de análisis y diseño de políticas públicas. Bogotá: Universidad Externado de Colombia / Edición Kindle.

Huffpost. (9 de octubre de 2018). Estas son las 10 empresas que más generan plástico a nivel mundial, según Greenpeace. Obtenido de Huffpost:bttps://wnw.buffingtonpost.com.mx/2018/10/09/empresas-que-mas-generan-basura-greenpeace_a_23554651/ [Verificado el 18 de diciembre de 2018]

Cumbre Pueblos. (18 de junio de 2018). Consumismo, sus causas y consecuencias. Obtenido de Cumbre Pueblos: https:// cumbrepuebloscop20.org/economia/ consumismo/ [Verificado el 18 de diciembre de 2018]

Reveles, C. (20 de agosto de 2018). Ciudad de México, al borde del colapso por la generación excesiva de basura. Obtenido de Animal Politico: bttps://mmw.animalpolitico.com/2018/08/cdmx-colapso-generacion-excesiva-basural _Verificado el 18 de diciembre de $2018]$

Mejía Vargas, A. C. (coordinador) (2017). Inventario de Residuos Sólidos 2016. Ciudad de México.

Sostenibilidad para Todos. (2017). Así es la lucha contra la obsolescencia programada. Obtenido de Acciona: https:// wmw. sostenibilidad.com/desarrollo-sostenible/asi-lucha-contra-obsolescencia-programadal [Verificado el 18 de diciembre de 2018] 